\title{
The effect of consuming boarfish (Capros aper) protein hydrolysate on glycated haemoglobin and BMI in overweight adults
}

\author{
William Crowe ${ }^{1}$, Judith Baird ${ }^{2}$, Christopher McLaughlin ${ }^{2}$, Padraigin Harnedy ${ }^{3}$, Dick Fitzgerald ${ }^{3}$, \\ Finbarr O'Harte ${ }^{2}$, Philip Allsopp ${ }^{2}$ and Emeir McSorley ${ }^{2}$ \\ ${ }^{1}$ Queen's University of Belfast, Belfast, Ireland, \\ ${ }^{2}$ Ulster University, Coleraine, Ireland and \\ ${ }^{3}$ University of Limerick, Limerick, Ireland
}

\section{Abstract}

Boarfish (Capros aper) is an underutilised pelagic fish species found in abundance in Irish waters. Research previously undertaken has shown a favourable effect of boarfish derived protein hydrolysate (BFH) consumption on glycaemic control in diabetic murine models. This study aims to investigate the effect of daily BFH consumption on glycated haemoglobin and body mass index (BMI) in overweight human participants.

A randomised parallel placebo-controlled human intervention study was undertaken to investigate the effect of consuming BFH $(3.5 \mathrm{~g} / \mathrm{day})$ for 12 weeks. Participants $(\mathrm{n}=40)$ that met the following inclusion criteria were recruited; aged between $18-65 \mathrm{years}$, nonsmoker, not currently pregnant or lactating, BMI $>25.0 \mathrm{~kg} / \mathrm{m}^{2}$, free from illness. Participants were randomly assigned to consume either BFH or cellulose capsules for 12 weeks. Measures taken at baseline and post-intervention (week 12) included fasting measures of: glycated haemoglobin, glucose, insulin, leptin, glucagon like peptide (GLP-1), adiponectin as well as lipid profile. Height and weight were also recorded at both timepoints. Analysis of covariance (ANCOVA) controlling for baseline values as well as other covariates (age \& BMI), was used to compare differences between groups over time.

Consumption of BFH (3.5g/day) for 12 weeks had no significant effect on glucose, insulin, leptin, glucagon like peptide (GLP-1), adiponectin, high density lipoprotein (HDL), total cholesterol, triglycerides, low density lipoprotein compared with control. No significant difference in glycated haemoglobin change over time $(\mathrm{P}=0.123)$ was noted following consumption of placebo $(2.5 \%$ increase $)$ in comparison to those consuming the boarfish treatment (1.5\% reduction).

This study showed that $3.5 \mathrm{~g}$ of $\mathrm{BFH}$ per day did not elicit an effect on any of the markers of metabolic health. Previous studies investigating the effect of protein consumption on metabolic health have used higher concentrations than were utilized in this study, however using similar concentrations in this study was not plausible given the lack of organoleptic acceptability of the BFH. Addressing the organoleptic properties of $\mathrm{BFH}$ may enable future studies to increase the dose of $\mathrm{BFH}$ to a level that may be more efficacious than the current study.

\section{Conflict of Interest}

no 